\title{
Topp-Leone Gompertz Distribution: Properties and Applications
}

\author{
Lawrence Chukwudumebi Nzei ${ }^{1}$, Joseph Thomas Eghwerido*2, and Nosakhare \\ EKHOSUEHI ${ }^{1}$ \\ ${ }^{1}$ Department of Statistics, University of Benin, Benin City, Edo State, Nigeria \\ ${ }^{2}$ Department of Mathematics, Federal University of Petroleum Resources, Effurun, Delta State, Nigeria
}

\begin{abstract}
This paper proposes the Topp-Leone Gompertz distribution; an extension of the Gompertz distribution for modeling real life time data. The new model is obtained by transforming the cumulative distribution function of the Gompertz random variable, while taking the Topp-Leone as the generator. Some statistical properties of the new distribution are derived. Maximum likelihood estimates of model parameters are also derived. A Monte Carlo simulation study is carried out to examine the accuracy of the maximum likelihood estimate of the distribution parameters. Two real data sets are used to illustrate the applicability of the new distribution, and the results show that the new distribution outperforms some related lifetime distributions.
\end{abstract}

Keywords generalized Gompertz; Gompertz-Lindley; linear representation.

\section{Introduction}

The Gompertz probability distribution is a continuous probability distribution introduced by Gompertz (1825). The Gompertz distribution is used to study the nature of human mortality by determining the value of life contingencies. The Gompertz distribution has been applied in several areas of statistics where survival time is required. Tjørve and Tjørve (2017) gave a detail review of the Gompertz distribution. The cumulative distribution function (CDF) of the Gompertz distribution is given as

$$
F(x)=1-\exp \left[\frac{\beta}{\alpha}\left(1-e^{\alpha x}\right)\right], \quad x>0, \alpha>0, \beta>0 .
$$

The corresponding probability distribution function (PDF) is given as

$$
f(x)=\beta e^{\alpha x} \exp \left[\frac{\beta}{\alpha}\left(1-e^{\alpha x}\right)\right], \quad x>0, \alpha>0, \beta>0 .
$$

The major shortfall of Gompertz distribution in lifetime data analysis is that the hazard rate function (hrf) is increasing for all parameter values. In order to accommodate flexibility into the hazard rate function. El-Gohary et al. (2013) introduced the generalized Gompertz distribution with increasing, constant, decreasing or bathtub curve failure rate. This property makes the generalized Gompertz distribution a better model in survival analysis than the classical Gompertz distribution. Ali et al. (2014) proposed the beta Gompertz distribution with flexibility shown in the increasing, decreasing and bathtub shape properties of the hazard rate function. Benkhelifa (2017) proposed the beta generalized Gompertz distribution whose hazard rate function has constant, increasing, decreasing or bathtub curve shape. The Modified beta Gompertz distribution

\footnotetext{
*Corresponding author. Email: eghwerido.joseph@fupre.edu.ng.
} 
proposed in Elbatal et al. (2019) has increasing, decreasing and bathtub hazard rate function. Others are J. W Wu et al. (2004), Marshall et al. (1983), Ohishi (2009), El-Damcese et al. (2015) among others.

In the recent years, statisticians have developed several methods for generating new distributions that are more flexibility. These new method have been used in literature to extend the Gompertz distribution. Khan et al. (2017) introduced the transmuted generalized Gompertz distribution using the concept of Transmuted-G family. Chukwu and Ogunde (2016) introduced the Kumuaraswamy Gompertz-Makeham distribution. Benkhelifa (2016) proposed the MarshallOlkin extended generalized Gompertz distribution using Marshall and Olkin (1997) method. Mohamed et al. (2019) considered the properties and applications of the alpha power transformation family introduced by Mahdavi and Kundu (2017), Eghwerido et al. (2020) proposed the alpha power Gompertz distribution and many others.

In this paper, we introduce a new distribution model called Topp-Leone Gompertz (TLGz) distribution and some of its statistical properties which includes, quantile function, moment, moment generating function, ordered statistics and Renyi entropy. The method of maximum likelihood estimation is used to estimate the parameters of the TLGz distribution. Finally, the flexibility and efficiency of the TLGz distribution over some existing ones are illustrated.

\section{The Topp-Leone Gompertz Distribution}

This section introduces the Topp-Leone Gompertz (TLGz) distribution using the idea of ToppLeone generated family of distributions introduced by Al-Shomrani et al. (2016).

Let $Y$ be a random variable of the Topp-Leone distribution with parameter $\theta$ denoted by $Y \sim T L(\theta)$. Then, the CDF and PDF of the distribution are expressed as

$$
G_{T L}(y)=y^{\theta}(2-y)^{\theta}, \quad 0<y<1, \theta>0
$$

and

$$
g_{T L}(y)=2 \theta y^{\theta-1}(1-y)(2-y)^{\theta-1}, \quad 0<y<1, \theta>0 .
$$

If $X$ is a continuous random variable with CDF $F(x)$, then the Topp-Leone generated (TL$\mathrm{G})$ family of distribution, $G(x)$ has the CDF defined as

$$
G(x)=[F(x)]^{\theta}[2-F(x)]^{\theta}=\left[1-(\bar{F}(x))^{2}\right]^{\theta}, \quad x>0, \theta>0 .
$$

The corresponding PDF, $g(x)$ is written as

$$
g(x)=2 \theta f(x) \bar{F}(x)[F(x)]^{\theta-1}[2-F(x)]^{\theta-1}=2 \theta f(x) \bar{F}(x)\left[1-(\bar{F}(x))^{2}\right]^{\theta-1},
$$

where $F(x)$ is the CDF of the baseline distribution, $\bar{F}(x)=1-F(x)$ and $f(x)=\frac{d F(x)}{d x}$ is the PDF of the baseline distribution. Substituting Equation (1) into Equation (5), we obtain the $\mathrm{CDF}$ of the Topp-Leone Gompertz (TLGz) distribution as

$$
G(x)=\left\{1-\exp \left[\frac{2 \beta}{\alpha}\left(1-e^{\alpha x}\right)\right]\right\}^{\theta}, \quad \beta>0, \alpha>0, \theta>0, x>0 .
$$




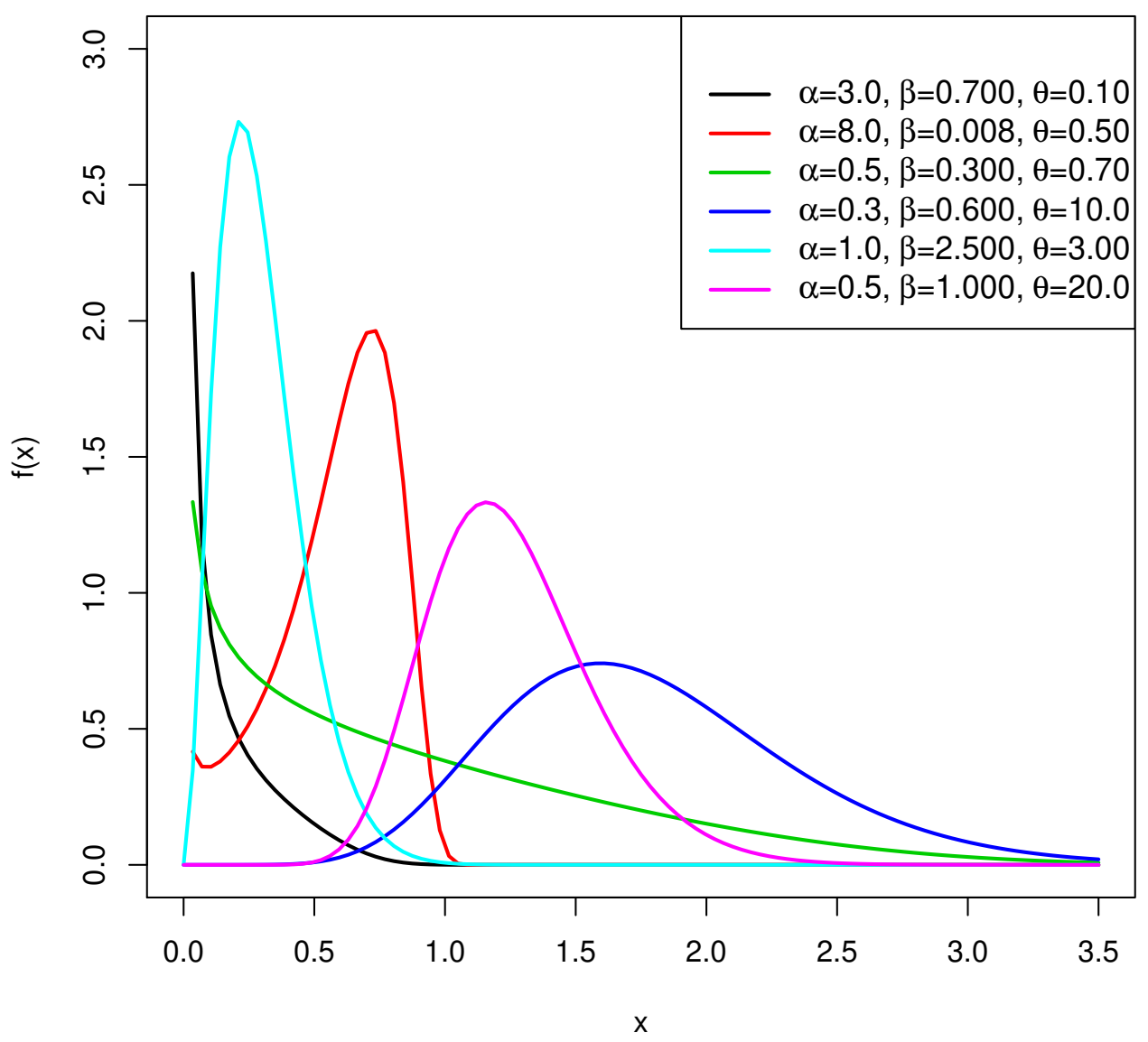

Figure 1: Plots of TLGz density function for different parameter values.

The corresponding probability density function is obtained by substituting Equation (2) into Equation (6) as;

$$
g(x)=2 \beta \theta e^{\alpha x} \exp \left[\frac{2 \beta}{\alpha}\left(1-e^{\alpha x}\right)\right]\left\{1-\exp \left[\frac{2 \beta}{\alpha}\left(1-e^{\alpha x}\right)\right]\right\}^{\theta-1}, \quad \beta>0, \alpha>0, \theta>0, x>0 .
$$

Figure 1 shows the plots of the TLGz density for some fixed parameter values. These density plots show that the TLGz distribution can be unimodal, right skewed and decreasing.

\subsection{The Survival and Hazard Rate Function}

The reliability function of the TLGz distribution is given as

$$
S(x)=1-G(x)=1-\left\{1-\exp \left[\frac{2 \beta}{\alpha}\left(1-e^{\alpha x}\right)\right]\right\}^{\theta} .
$$

The hazard rate function is obtained as

$$
h(x)=\frac{f(x)}{S(x)}=\frac{2 \beta \theta e^{\alpha x} \exp \left[\frac{2 \beta}{\alpha}\left(1-e^{\alpha x}\right)\right]\left\{1-\exp \left[\frac{2 \beta}{\alpha}\left(1-e^{\alpha x}\right)\right]\right\}^{\theta-1}}{1-\left\{1-\exp \left[\frac{2 \beta}{\alpha}\left(1-e^{\alpha x}\right)\right]\right\}^{\theta}} .
$$




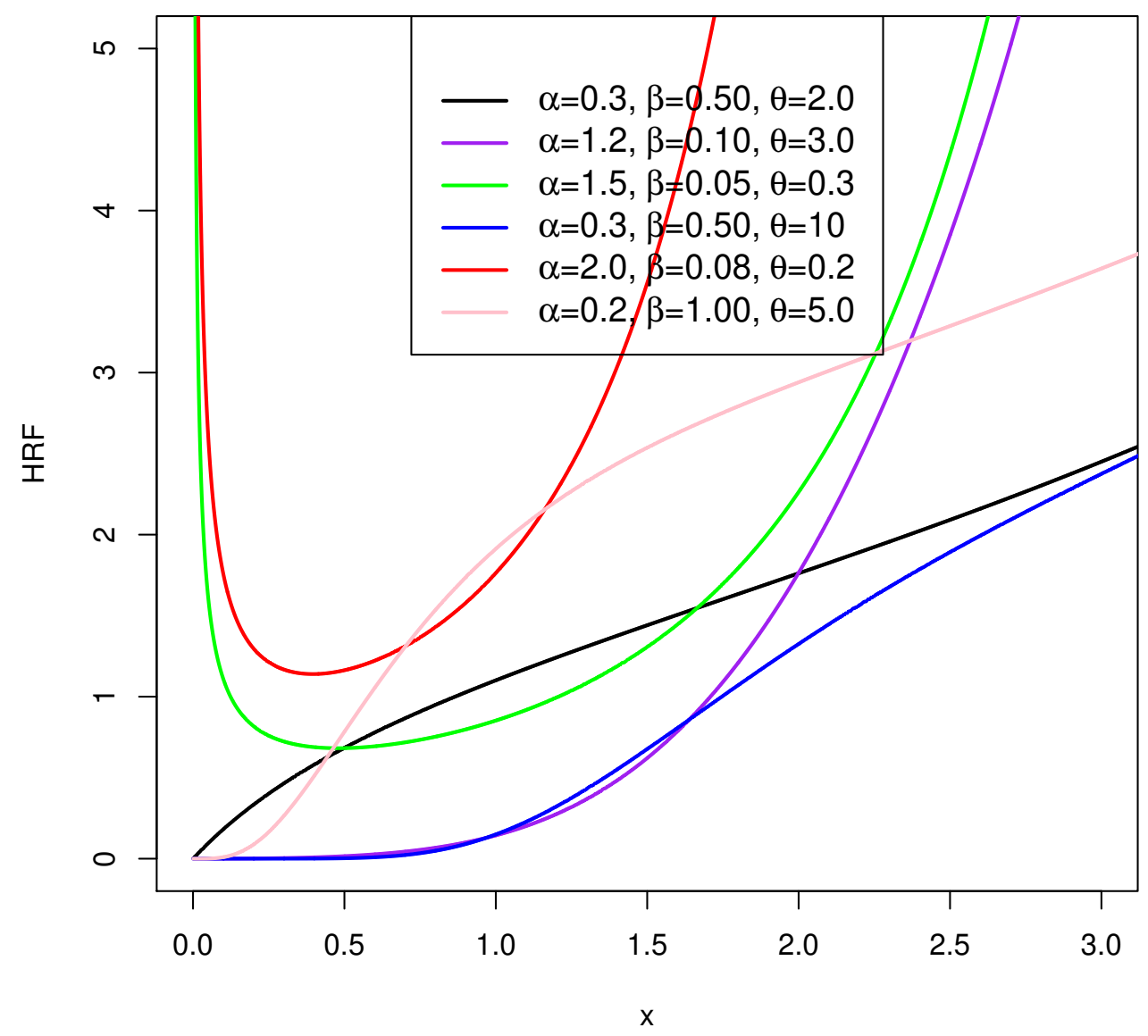

Figure 2: Plots of TLGz hazard rate function for different parameter values.

Figure 2 shows the plots of the TLGz hazard rate function for some fixed parameter values. The hazard rate function plots show that the TLGz distribution can be bathtub, concave and convex increasing.

\subsection{Expansion of TLGz CDF and PDF}

Using the binomial series expansion defined as $(1-z)^{c}=\sum_{i=0}^{\infty}\left(\begin{array}{c}c \\ i\end{array}\right)(-1)^{i} z^{i}$, then we can express the CDF of TLGz distribution in (3) as

$$
\begin{aligned}
G(x) & =\sum_{i=0}^{\infty}\left(\begin{array}{l}
\theta \\
i
\end{array}\right)(-1)^{i} e^{\frac{2 \beta}{\alpha} i} e^{-\frac{2 \beta}{\alpha} i e^{\alpha x}} \\
& =\sum_{i=0}^{\infty} \sum_{j=0}^{\infty}\left(\begin{array}{l}
\theta \\
i
\end{array}\right)\left(\frac{2 \beta}{\alpha} i\right)^{j} \frac{(-1)^{i+j}}{j !} e^{\frac{2 \beta}{\alpha} i} e^{j \alpha x},
\end{aligned}
$$

since $e^{t x}=\sum_{i=0}^{\infty} \frac{(t x)^{i}}{i !}$ 
Similarly, the PDF of TLGz distribution can be represented as

$$
\begin{aligned}
g(x) & =\sum_{i=0}^{\infty}\left(\begin{array}{c}
\theta-1 \\
i
\end{array}\right)(-1)^{i} e^{\alpha x} e^{\frac{2 \beta}{\alpha}(1+i)} e^{-\frac{2 \beta}{\alpha}(1+i) e^{\alpha x}} \\
& =2 \beta \theta \sum_{i=0}^{\infty} \sum_{j=0}^{\infty}\left(\begin{array}{c}
\theta-1 \\
i
\end{array}\right)\left(\frac{2 \beta}{\alpha}\right)^{j} e^{\frac{2 \beta}{\alpha}(1+i)} \frac{(-1)^{i+j}(1+i)^{j}}{j !} e^{\alpha(i+j) x} .
\end{aligned}
$$

\section{Mathematical Properties}

In this section, some mathematical properties of the newly proposed Topp-Leone Gompertz distribution were examined. These properties include quantile, the raw moments, moment generating function, probability weighted moments, entropies and the distribution of order statistics.

\subsection{Quantile Function}

The quantile function is obtained by inverting the CDF of the TLGz distribution. Given that $0<u<1$, then the quantile function of a random variable $X$ is given as

$$
Q(u)=\frac{1}{\alpha} \log \left\{1-\frac{\alpha}{2 \beta} \log \left(1-u^{\frac{1}{\theta}}\right)\right\} .
$$

Hence, for $u=0.5$, we obtain the median of the distribution as

$$
Q(0.5)=\frac{1}{\alpha} \log \left\{1-\frac{\alpha}{2 \beta} \log \left(1-0.5^{\frac{1}{\theta}}\right)\right\} .
$$

The quantile function, in Equation (13) is used to simulate random numbers $X=u$ from TLGz distribution where $U$ is a random variable following the uniform distribution over $(0,1)$.

The skewness and kurtosis of the TLGz can also determine from $Q(u)$ using the Bowley $(B)$ skewness and Moors kurtosis $(M)$ respectively defined as

and

$$
B=\frac{Q\left(\frac{3}{4}\right)+Q\left(\frac{1}{4}\right)-2 Q\left(\frac{1}{2}\right)}{Q\left(\frac{3}{4}\right)-Q\left(\frac{1}{4}\right)}
$$

$$
M=\frac{Q\left(\frac{7}{8}\right)-Q\left(\frac{3}{8}\right)-Q\left(\frac{5}{8}\right)+Q\left(\frac{1}{8}\right)}{Q\left(\frac{3}{4}\right)-Q\left(\frac{1}{4}\right)}
$$

\subsection{Moments}

Given that $r$ is a non-negative integer, then the $r^{\text {th }}$ moment about the origin of a continuous random variable $X$ is defined by $\mu_{r}^{\prime}=E\left(X^{r}\right)=\int_{-\infty}^{\infty} x^{r} g(x) d x$. Using the PDF as expressed in Equation (12), we have

$$
\mu_{r}^{\prime}=2 \beta \theta \sum_{i=0}^{\infty} \sum_{j=0}^{i} \sum_{k=0}^{j}\left(\begin{array}{c}
\theta-1 \\
i
\end{array}\right)\left(\begin{array}{l}
j \\
k
\end{array}\right)\left(\frac{2 \beta}{\alpha}\right)^{j} \frac{(-1)^{i+k}(1+i)^{j}}{j !} \int_{0}^{\infty} x^{r} e^{\alpha(1+k) x} d x .
$$

By using change of variable as $u=-\alpha(1+k) x$ and $x=\frac{-u}{\alpha(1+k)}$, we obtain

$$
\mu_{r}^{\prime}=2 \beta \theta \sum_{i=0}^{\infty} \sum_{j=0}^{i} \sum_{k=0}^{j}\left(\begin{array}{c}
\theta-1 \\
i
\end{array}\right)\left(\begin{array}{l}
j \\
k
\end{array}\right)\left(\frac{2 \beta}{\alpha}\right)^{j} \frac{(-1)^{i+k}(1+i)^{j}}{j !}\left[\frac{-u}{\alpha(1+k)}\right]^{r+1} \int_{0}^{\infty} u^{r} e^{u} d u
$$




\subsection{Moment Generating Function}

The moment generating function of a random variable $X$ is the formula (function) used to find the moments of the random variable. Mathematical definition of moment generating function of a random variable $X$ with $\mathrm{PDF} g(x)$ is defined as $M_{X}(z)=E\left(e^{z x}\right)=\int_{-\infty}^{\infty} e^{z x} g(x) d x$. Thus, by Equation (12), we have the TLGz distribution expressed as

$$
M_{X}(z)=E\left(e^{z x}\right)=2 \beta \theta \sum_{i=0}^{\infty} \sum_{j=0}^{i} \sum_{k=0}^{j}\left(\begin{array}{c}
\theta-1 \\
i
\end{array}\right)\left(\begin{array}{l}
j \\
k
\end{array}\right)\left(\frac{2 \beta}{\alpha}\right)^{j} \frac{(-1)^{i+k}}{j !} \int_{0}^{\infty} e^{[z+\alpha(1+k)] x} d x .
$$

Using change of variable as $u=-\alpha(1+k) x$ and $x=\frac{-u}{\alpha(1+k)}$, then

$$
\begin{aligned}
E\left(e^{z x}\right) & =2 \beta \theta \sum_{i=0}^{\infty} \sum_{j=0}^{i} \sum_{k=0}^{j}\left(\begin{array}{c}
\theta-1 \\
i
\end{array}\right)\left(\begin{array}{c}
j \\
k
\end{array}\right)\left(\frac{2 \beta}{\alpha}\right)^{j} \frac{(-1)^{i+k+1}(1+k)^{j}}{j ![z+\alpha(1+k)]} \int_{0}^{\infty} e^{u} d u . \\
& =2 \beta \theta \sum_{i=0}^{\infty} \sum_{j=0}^{i} \sum_{k=0}^{j}\left(\begin{array}{c}
\theta-1 \\
i
\end{array}\right)\left(\begin{array}{l}
j \\
k
\end{array}\right)\left(\frac{2 \beta}{\alpha}\right)^{j} \frac{(-1)^{i+k+1}(1+k)^{j}}{j ![z+\alpha(1+k)]} .
\end{aligned}
$$

\subsection{Renyi Entropy}

Entropy is the study of uncertainty with respect to a random variable. If $X$ is a random variable under study with PDF $g(x)$, then the Renyi entropy of $X$ is defined as

$$
I_{R}(s)=\frac{1}{1-s} \log \phi(s), \quad s>0
$$

where,

$$
\phi(s)=\int_{0}^{\infty} g^{s}(x) d x=(2 \beta \theta)^{s} \int_{0}^{\infty} e^{s \alpha x} e^{\frac{2 \beta s}{\alpha}\left(1-e^{\alpha x}\right)}\left\{1-\exp \left[\frac{2 \beta}{\alpha}\left(1-e^{\alpha x}\right)\right]\right\}^{s(\theta-1)} d x .
$$

However, using binomial expansion and power series expansion, we have

$$
\phi(s)=(2 \beta \theta)^{s} \sum_{i=0}^{\infty} \sum_{j=0}^{i} \sum_{k=0}^{j}\left(\begin{array}{c}
\theta-1 \\
i
\end{array}\right)\left(\begin{array}{l}
j \\
k
\end{array}\right)\left(\frac{2 \beta}{\alpha}\right)^{k} \frac{(-1)^{i+k}(s+1)^{j}}{j !} \int_{0}^{\infty} e^{k \alpha x} d x .
$$

Thus,

$$
I_{R}(s)=\frac{1}{1-s} \log \left\{(2 \beta \theta)^{s} \sum_{i=0}^{\infty} \sum_{j=0}^{i} \sum_{k=0}^{j}\left(\begin{array}{c}
\theta-1 \\
i
\end{array}\right)\left(\begin{array}{l}
j \\
k
\end{array}\right)\left(\frac{2 \beta}{\alpha}\right)^{k} \frac{(-1)^{i+k+1}(s+1)^{j}}{j ! k \alpha} \int_{0}^{\infty} e^{k \alpha x} d x\right\}
$$

\subsection{Distribution of Order Statistics}

Suppose $X_{1}, \cdots, X_{n}$ are random variables from TLGz distribution and $X_{1: n}, \cdots, X_{n: n}$ are the corresponding order statistics of the random sample. Then the probability density function of 
the $k^{\text {th }}$ order statistics $X=X_{k: n}, k=1, \cdots, n$, denoted by $f_{k: n}(x)$, for $1 \leq x \leq n$ is defined as

$$
\begin{aligned}
f_{k: n}(x) & =\frac{n !}{(k-1) !(n-k) !} f(x) F^{k-1}(x)[1-F(x)]^{n-k} \\
& =\frac{n !}{(k-1) !(n-k) !} \sum_{i=0}^{n-k}\left(\begin{array}{c}
n-k \\
i
\end{array}\right)(-1)^{i} f(x)[1-F(x)]^{k+i-1},
\end{aligned}
$$

where $f(x)$ and $F(x)$ are the $\mathrm{PDF}$ and $\mathrm{CDF}$ of the random variable $\mathrm{X}$, respectively. Now, Substituting Equation (7) and Equation (8) into Equation (18), we obtain the density function of the order statistics for TLGz distribution as

$$
\begin{aligned}
f_{k: n}(x) & =\frac{2 \beta \theta n !}{(k-1) !(n-k) !} \sum_{i=0}^{n-k} \sum_{j=0}^{\theta+k+i-2} \sum_{m=0}^{j} \sum_{p=0}^{m}\left(\begin{array}{c}
n-k \\
i
\end{array}\right)\left(\begin{array}{c}
\theta+k+i-2 \\
j
\end{array}\right)\left(\frac{2 \beta}{\alpha}\right)^{k} \\
& \times \frac{(-1)^{i+m+1}[\alpha(1+i)]^{p} x^{p}}{m ! p !} .
\end{aligned}
$$

\section{Statistical Inference}

In this section, we estimate the parameters of the proposed Topp-Leone Gompertz distribution by using the maximum likelihood estimate and a simulation study is performed to investigate the efficiency of the maximum likelihood estimates (MLEs) of the proposed Topp-Leone Gompertz distribution.

\subsection{Maximum Likelihood Estimation}

In this section, the estimation of the TLGz parameters through the maximum likelihood estimation method was obtained. Suppose a random sample $x_{1}, x_{2}, x_{3}, \cdots, x_{n}$ of size $\mathrm{n}$ follows a TLGz distribution, then the maximum likelihood function is given as

$$
L=\prod_{i=1}^{n}\left\{2 \beta \theta e^{\alpha x_{i}} e^{\frac{2 \beta}{\alpha}\left(1-e^{\alpha x_{i}}\right)}\left[1-e^{\frac{2 \beta}{\alpha}\left(1-e^{\alpha x_{i}}\right)}\right]^{\theta-1}\right\}
$$

and the log-likelihood function $\ell_{n}=\log (L)$ becomes

$$
\ell_{n}=n \log (2 \beta \theta)+\alpha \sum_{i=1}^{n} x_{i}+\frac{2 \beta}{\alpha} \sum_{i=1}^{n} \log \left(1-e^{\alpha x_{i}}\right)+(\theta-1) \sum_{i=1}^{n} \log \left[1-e^{\frac{2 \beta}{\alpha}\left(1-e^{\alpha x_{i}}\right)}\right] .
$$

The partial derivatives of Equation (20) with respect to the parameters $\alpha, \beta$ and $\theta$ are

$$
\begin{aligned}
\frac{\partial \ell_{n}}{\partial \alpha}= & \sum_{i=1}^{n} x_{i}-\frac{2 \beta}{\alpha} \sum_{i=1}^{n}\left\{x_{i} e^{\alpha x_{i}}+\frac{1}{\alpha}\left(1-e^{\alpha x_{i}}\right)\right\} \\
& -(\theta-1) \sum_{i=1}^{n} \frac{\frac{2 \beta}{\alpha}\left\{\frac{1}{\alpha}\left(1-e^{\alpha x_{i}}\right)+x_{i} e^{\alpha x_{i}}\right\} e^{\frac{2 \beta}{\alpha}\left(1-e^{\alpha x_{i}}\right)}}{1-e^{\frac{2 \beta}{\alpha}\left(1-e^{\alpha x_{i}}\right)}} \\
\frac{\partial \ell_{n}}{\partial \beta}= & \frac{n}{\beta}+\frac{2}{\alpha} \sum_{i=1}^{n}\left(1-e^{\alpha x_{i}}\right)-(\theta-1) \sum_{i=1}^{n} \frac{\frac{2}{\alpha}\left(1-e^{\alpha x_{i}}\right) e^{\frac{2 \beta}{\alpha}\left(1-e^{\alpha x_{i}}\right)}}{1-e^{\frac{2 \beta}{\alpha}\left(1-e^{\alpha x_{i}}\right)}}
\end{aligned}
$$




$$
\frac{\partial \ell_{n}}{\partial \theta}=\frac{n}{\theta}+\sum_{i=1}^{n} \log \left[1-e^{\frac{2 \beta}{\alpha}\left(1-e^{\alpha x_{i}}\right)}\right] .
$$

The $\operatorname{MLEs}(\hat{\alpha}, \hat{\beta}, \hat{\theta})$ for $(\alpha, \beta, \theta)$ are obtained by setting the score function to zero, $U(\Phi)=0$ and solve the system of equation simultaneously where $U(\Phi)=\left(\frac{\partial \ell_{n}}{\partial \alpha}, \frac{\partial \ell_{n}}{\partial \beta}, \frac{\partial \ell_{n}}{\partial \theta}\right)^{T}=0$.

The non-linear system of equation is solved iteratively using $\mathrm{R}$ statistical package to obtain the MLE for the unknown parameters. The Newton-Raphson iterative method can easily be used since the second partial derivative exist. This numerical estimates of $\hat{\Phi}=(\hat{\alpha}, \hat{\beta}, \hat{\theta})$ are obtained with $\mathrm{R}$ statistical package.

The approximate confidence interval, confidence region and testing hypothesis of $(\alpha, \beta, \theta)$ can be determined using the MLEs $(\hat{\alpha}, \hat{\beta}, \hat{\theta})$.

\subsection{Simulation Study}

In this section, we carry out a simulation study to investigate the performance and accuracy of maximum likelihood estimates of TLGz distribution model parameters for various combinations of 6 sample sizes considering two sets of parameter values. The quantile function was used to generate random data from TLGz distribution. In each simulation, 10,000 samples of sizen =

Table 1: Simulation Results: - Mean, Bias and RMSE 10,000 MLEs of TLGz for some fixed parameter values.

\begin{tabular}{|c|c|c|c|c|c|c|c|c|}
\hline \multirow[b]{2}{*}{$n$} & \multicolumn{4}{|c|}{ I } & \multicolumn{4}{|c|}{ II } \\
\hline & Parameter & Mean & Bias & RMSE & Parameter & Mean & Bias & RMSE \\
\hline \multirow[t]{3}{*}{50} & $\alpha=1.3$ & 1.3669 & 0.0669 & 0.5425 & $\alpha=3.0$ & 3.0908 & 0.0908 & 1.0305 \\
\hline & $\beta=0.5$ & 0.5491 & 0.0491 & 0.2784 & $\beta=0.7$ & 0.7912 & 0.0912 & 0.4251 \\
\hline & $\theta=2.0$ & 2.2574 & 0.2574 & 1.0533 & $\theta=1.5$ & 1.6868 & 0.1868 & 0.7370 \\
\hline \multirow[t]{3}{*}{75} & $\alpha=1.3$ & 1.3354 & 0.0354 & 0.4318 & $\alpha=3.0$ & 3.0434 & 0.0434 & 0.8416 \\
\hline & $\beta=0.5$ & 0.5352 & 0.0352 & 0.2167 & $\beta=0.7$ & 0.7670 & 0.0670 & 0.3415 \\
\hline & $\theta=2.0$ & 2.1651 & 0.1651 & 0.7286 & $\theta=1.5$ & 1.6236 & 0.1236 & 0.5203 \\
\hline \multirow[t]{3}{*}{100} & $\alpha=1.3$ & 1.3278 & 0.0278 & 0.3706 & $\alpha=3.0$ & 3.0341 & 0.0341 & 0.7342 \\
\hline & $\beta=0.5$ & 0.5244 & 0.0244 & 0.1796 & $\beta=0.7$ & 0.7485 & 0.0485 & 0.2814 \\
\hline & $\theta=2.0$ & 2.1198 & 0.1198 & 0.5751 & $\theta=1.5$ & 1.5911 & 0.0911 & 0.4098 \\
\hline \multirow[t]{3}{*}{150} & $\alpha=1.3$ & 1.3239 & 0.0239 & 0.3033 & $\alpha=3.0$ & 3.0306 & 0.0306 & 0.5976 \\
\hline & $\beta=0.5$ & 0.5141 & 0.0141 & 0.1518 & $\beta=0.7$ & 0.7287 & 0.0287 & 0.2308 \\
\hline & $\theta=2.0$ & 2.0738 & 0.0738 & 0.4746 & $\theta=1.5$ & 1.5544 & 0.0544 & 0.3248 \\
\hline \multirow[t]{3}{*}{200} & $\alpha=1.3$ & 1.3158 & 0.0158 & 0.2595 & $\alpha=3.0$ & 3.0305 & 0.0306 & 0.5196 \\
\hline & $\beta=0.5$ & 0.5114 & 0.0114 & 0.1224 & $\beta=0.7$ & 0.7190 & 0.0190 & 0.1896 \\
\hline & $\theta=2.0$ & 2.0554 & 0.0554 & 0.3734 & $\theta=1.5$ & 1.5337 & 0.0357 & 0.2573 \\
\hline \multirow[t]{3}{*}{300} & $\alpha=1.3$ & 1.3108 & 0.0108 & 0.2218 & $\alpha=3.0$ & 3.0206 & 0.0206 & 0.4349 \\
\hline & $\beta=0.5$ & 0.5070 & 0.0070 & 0.1045 & $\beta=0.7$ & 0.7114 & 0.0114 & 0.1560 \\
\hline & $\theta=2.0$ & 2.0314 & 0.0314 & 0.3003 & $\theta=1.5$ & 1.5208 & 0.0208 & 0.2055 \\
\hline \multirow[t]{3}{*}{500} & $\alpha=1.3$ & 1.3021 & 0.0021 & 0.1677 & $\alpha=3.0$ & 3.0080 & 0.0080 & 0.3366 \\
\hline & $\beta=0.5$ & 0.5062 & 0.0062 & 0.0816 & $\beta=0.7$ & 0.7088 & 0.0088 & 0.1224 \\
\hline & $\theta=2.0$ & 2.0253 & 0.0253 & 0.2415 & $\theta=1.5$ & 1.5154 & 0.0154 & 0.1633 \\
\hline
\end{tabular}


$50,75,100,150,200,300$ was generated for some sets of parameter values $I: \alpha=1.3, \beta=0.5, \theta=$ 2.0 and $I I: \alpha=3.0, \beta=0.7, \theta=1.5$. R Statistical software was used to compute the following quantities in this simulation study:

$$
\begin{gathered}
\text { (i) Mean }=\frac{1}{10,000} \sum_{i=1}^{10,000} \hat{\Phi}, \\
\text { (ii) Bias }=\frac{1}{10,000} \sum_{i=1}^{10,000}(\hat{\Phi}-\Phi),
\end{gathered}
$$

and

$$
\text { (iii) Root Mean Square Error }(R M S E)=\sqrt{\frac{1}{10,000} \sum_{i=1}^{10,000}(\hat{\Phi}-\Phi)^{2}} \text {, }
$$

where $\hat{\Phi}=(\hat{\alpha}, \hat{\beta}, \hat{\theta})$ is an estimate of $\Phi=(\alpha, \beta, \theta)$.

From the simulation results (see Table 1 ), it is clearly seen that as the sample size increases, the estimated mean gets closer to the true parameter value. The biases decrease and the RMSEs decrease toward zero. This shows the efficiency of the maximum likelihood estimation method for the parameters of the Topp-Leone Gompertz (TLGz) distribution model.

\section{Applications}

In this section, applications to lifetime data sets were illustrated to examine the efficiency and superiority of the TLGz distribution in modeling real data practice. The TLGz distribution model is fitted to the selected data sets and compared to the fits of Kumaraswamy Gompertz (KGz), Weibull Gompertz (WGz), Weibull Frechet (WFr) and Transmuted Gompertz (TGz) distributions.

The negative log-likelihood $(-\ell)$, Akaike Information Criterion $(A I C)$, Bayesian Information Criterion $(B I C)$, Cramer-von Mises $\left(W^{*}\right)$ and Anderson-Darling $\left(A^{*}\right)$ values are computed and compared. It follows that the model with the smallest criteria values has the best fit. Also, the Kolmogorov-Smirnov (KS) statistic and the p-value were also examined.

However, the values of $A I C, B I C, W^{*}$ and $A^{*}$ sometimes are influenced by the number of parameters in the given distribution. Therefore, the KS statistic and the p-value are sufficient criteria for comparing models. The model with the lowest KS value and highest $\mathrm{p}$-value has the best fit.

\subsection{Data Set 1}

The given data represents the breaking stress of carbon fibers (in Gba) as observed and reported by Nichols and Padgett (2006) as used in Efe-eyefia et al. (2020):

$3.70,2.74,2.73,2.50,3.60,3.11,3.27,2.87,1.47,3.11,3.56,4.42,2.41,3.19,3.22,1.69,3.28$, $3.09,1.87,3.15,4.90,1.57,2.67,2.93,3.22,3.39,2.81,4.20,3.33,2.55,3.31,3.31,2.85,1.25$, $4.38,1.84,0.39,3.68,2.48,0.85,1.61,2.79,4.70,2.03,1.89,2.88,2.82,2.05,3.65,3.75,2.43$, $2.95,2.97,3.39,2.96,2.35,2.55,2.59,2.03,1.61,2.12,3.15,1.08,2.56,1.80,2.53$ 
Table 2: The MLEs and S.Es (in parentheses) for Data Set 1 Parameter Estimates

\begin{tabular}{ccccc}
\hline Model & $\hat{\alpha}$ & $\hat{\beta}$ & $\hat{\theta}$ & $\hat{\lambda}$ \\
\hline TLGz & 3.3580 & 0.2047 & 0.3432 & \\
& $(1.0651)$ & $(0.0731)$ & $(0.1172)$ & 0.5484 \\
$\mathrm{KGz}$ & 1.9584 & 0.1609 & 0.9779 & $(0,00341)$ \\
& $(0.5356)$ & $(0.0175)$ & $(0.00343)$ & -0.2706 \\
$\mathrm{WGz}$ & 0.2403 & 2.7550 & 0.4887 & $(0.4400)$ \\
& $(3.8765)$ & $(2.4725)$ & 0.9008 & \\
$\mathrm{TGz}$ & 0.7346 & 0.0376 & $(0.0845)$ & \\
& $(0.1756)$ & $(0.0096)$ & & \\
\hline
\end{tabular}

Table 3: Summary Statistics for the Data Set 1

\begin{tabular}{lccccccc}
\hline Model & $-\ell$ & $A I C$ & $B I C$ & $W^{*}$ & $A^{*}$ & KS & p-value \\
\hline $\mathrm{TLGz}$ & 141.3899 & 288.7799 & 296.5954 & 0.0721 & 0.4266 & 0.06337 & 0.8114 \\
$\mathrm{KGz}$ & 146.9744 & 301.9488 & 312.3695 & 0.0741 & 0.5619 & 0.1221 & 0.1009 \\
$\mathrm{WGz}$ & 141.2633 & 290.5265 & 300.9472 & 0.0660 & 0.3872 & 0.0640 & 0.8064 \\
$\mathrm{TGz}$ & 147.0786 & 300.1573 & 307.9728 & 0.1262 & 1.0366 & 0.0801 & 0.5411 \\
\hline
\end{tabular}

\subsection{Data Set 2}

Data Set 2 consists of 63 observations of the strengths of $1.5 \mathrm{~cm}$ glass fibers obtained by workers at the UK National Physical Laboratory, reported by Smith and Naylor (1987) and used in Eghwerido et al. (2020), Eghwerido et al. (2020b), Eghwerido et al. (2020a), and Zelibe et al. (2019):

$0.55,0.74,0.77,0.81,0.84,0.93,1.04,1.11,1.13,1.24,1.25,1.27,1.28,1.29,1.30,1.36,1.39$, $1.42,1.48,1.48,1.49,1.49,1.50,1.50,1.51,1.52,1.53,1.54,1.55,1.55,1.58,1.59,1.60,1.61$, $1.61,1.61,1.61,1.62,1.62,1.63,1.64,1.66,1.66,1.66,1.67,1.68,1.68,1.69,1.70,1.70,1.73$, $1.76,1.76,1.77,1.78,1.81,1.82,1.84,1.84,1.89,2.00,2.01,2.24$.

Table 2 and Table 4 present the MLEs of the unknown parameters with the corresponding standard errors (S.Es) enclosed in parentheses for Data Set 1 and 2 respectively. Table 3 and

Table 4: The MLEs and S.Es (in parentheses) for Data Set 2 Parameter Estimates

\begin{tabular}{ccccc}
\hline Model & $\hat{\alpha}$ & $\hat{\beta}$ & $\hat{\theta}$ & $\hat{\lambda}$ \\
\hline TLGz & 1.7421 & 0.0224 & 2.7473 & \\
& $(0.6408)$ & $(0.0209)$ & $(0.5477)$ & 3.1153 \\
$\mathrm{KGz}$ & 1.5553 & 0.2144 & 0.0974 & $(0.0147)$ \\
& $(0.4226)$ & $(0.0484)$ & $(0.0181)$ & -0.0021 \\
$\mathrm{WGz}$ & 0.0317 & 3.2269 & 0.8327 & $(0.3602)$ \\
& $(0.0846)$ & $(1.3041)$ & $(0.5645)$ & 0.2130 \\
$\mathrm{WFr}$ & 3.6121 & 25.1859 & 0.1623 & $(\mathrm{NaN})$ \\
& $(18.9309)$ & $(\mathrm{NaN})$ & $(\mathrm{NaN})$ & \\
\hline
\end{tabular}


Table 5: Summary Statistics for the Data Set 2

\begin{tabular}{lccccccc}
\hline Model & $-\ell$ & $A I C$ & $B I C$ & $W^{*}$ & $A^{*}$ & KS & p-value \\
\hline TLGz & 14.1657 & 34.3314 & 40.7608 & 0.1699 & 0.9511 & 0.1353 & 0.1989 \\
$\mathrm{KGz}$ & 14.7378 & 37.4757 & 46.0482 & 0.1799 & 1.0097 & 0.1520 & 0.1086 \\
$\mathrm{WGz}$ & 14.4165 & 36.8331 & 45.4056 & 0.1808 & 1.0123 & 0.1387 & 0.1764 \\
$\mathrm{WFr}$ & 15.3980 & 38.7960 & 47.3685 & 0.2471 & 1.3565 & 0.1552 & 0.0960 \\
\hline
\end{tabular}

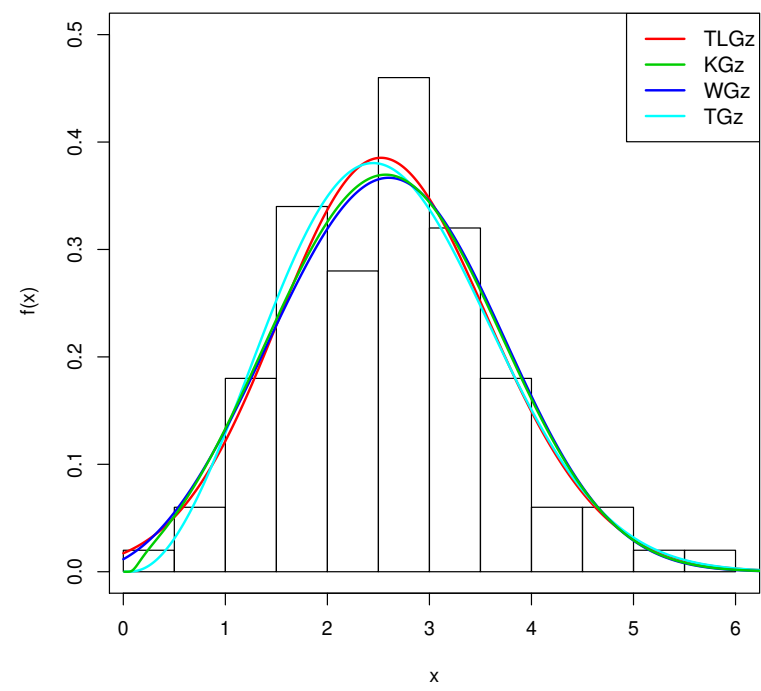

Figure 3: The fitted TLGz density and other densities for the first data.

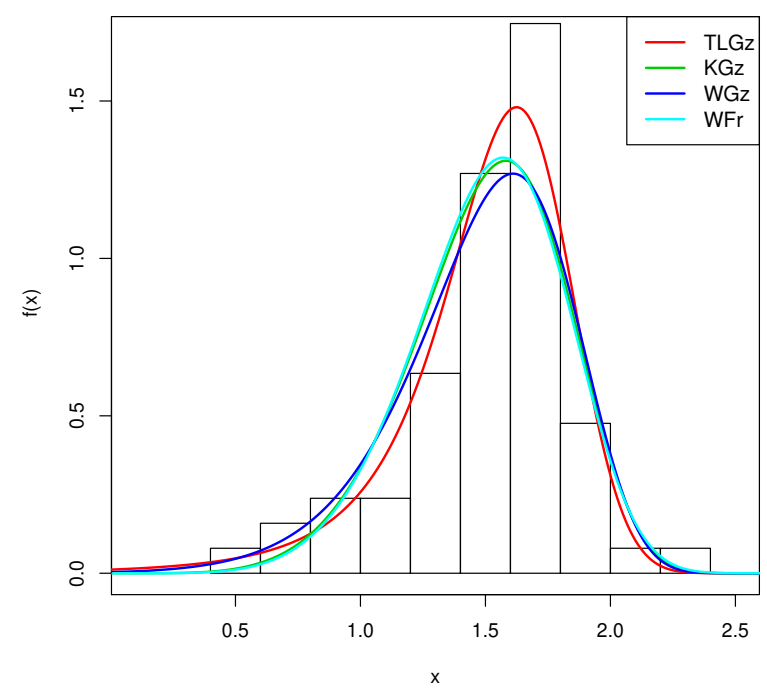

Figure 5: The fitted TLGz density and other densities for the second data.

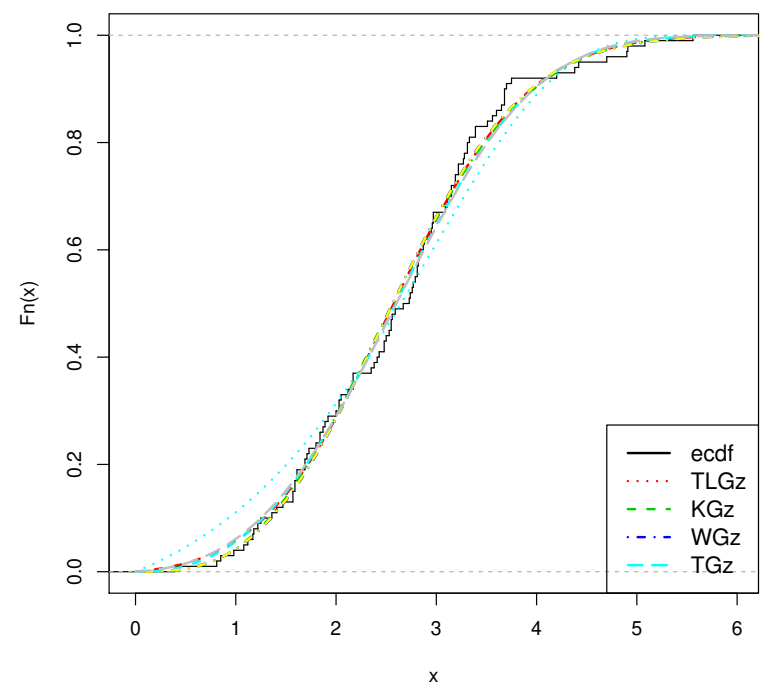

Figure 4: The fitted CDF of the TLGz density and other densities for the first data.

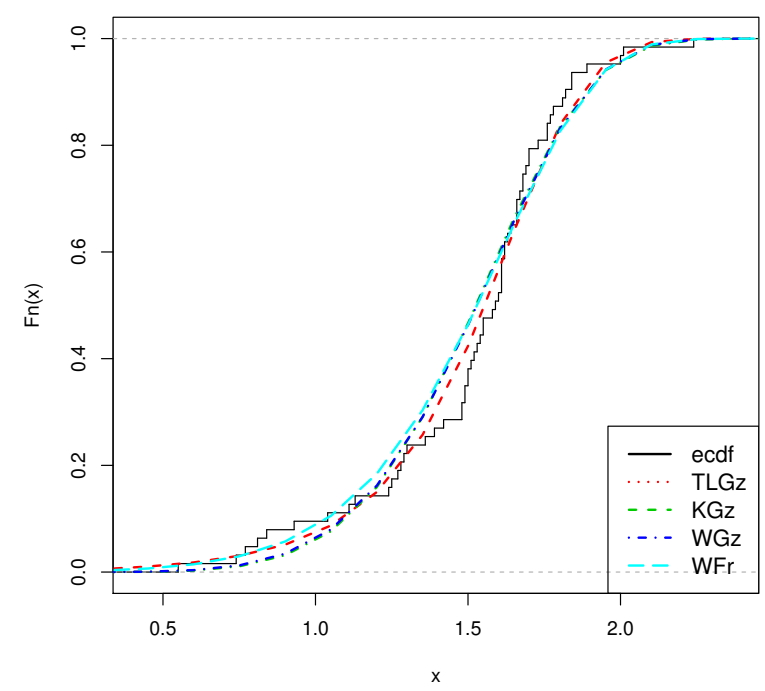

Figure 6: The fitted CDF of the TLGz density and other densities for the second data. 
Table 5 shows the statistics: $A I C, B I C, W^{*}, A^{*}, \mathrm{KS}$ and p-Values for all the models considered.

In Table 3 and Table 5, it is observed that TLGz has the highest p-Values and the lowest KS values for Data Set 1 and 2 respectively. This shows that TLGz distribution performs better in fitting these data sets.

However, the empirical density functions of the TLGz model compared with some densities are presented in Figure 3 and Figure 5 for Data Set 1 and Data Set 2 respectively. While Figure 4 and Figure 6 are the empirical cumulative distribution of the TLGz distribution compared with some CDFs. These plots indicated that the plot of TLGz model yields a better fit for both data sets.

\section{Concluding Remark}

This paper introduced a new distribution called "Topp-Leone Gompertz (TLGz) distribution"

using the Topp-Leone characterization introduced by Al-Shomrani et al. (2016). Some of its mathematical properties such as the quantile, moment, moment generating function, Renyi entropy and the distribution of order statistics were derived, studied and examined. The maximum likelihood estimates of the three parameters were obtained. Two data sets were fitted to the proposed model to illustrate the flexibility of TLGz distribution in life time analysis. The results shown that the TLGz model can be used as alternative model in life time analysis since is a strong competitor. More so, the newly proposed model could also be used to model life time survival processes where bathtub hazard rate is required. Furthermore, Bayesian reliability analysis for the proposed model could be a subject for further research for censored, Type 1 and Type 2 data.

\section{References}

Al-Shomrani A, Arif O, Shawky K, Hanif S, Shahbaz MQ (2016). Topp-Leone family of distributions: Some properties and application. Pakistan Journal of Statistics and Operation Research, 12(3): 443-451.

Ali AJ, Saeid T, Morad A (2014). The Beta-Gompertz distribution. Revista Colombiana de Estadistica, 37(1): 141-158.

Benkhelifa L (2016). The Marshall-Olkin extended generalized Gompertz distribution. Journal of Data Science, 15(2): 239-266.

Benkhelifa L (2017). The Beta generalized Gompertz distribution. Applied Mathematical Modelling, 52: 341-357.

Chukwu AU, Ogunde AA (2016). On Kumaraswamy Gompertz Makeham distribution. American Journal of Mathematics and Statistics, 6(3): 122-127.

Efe-eyefıa E, Thomas J, Zelıbe SC (2020). Theoretical analysis of the Weibull alpha power inverted exponential distribution: Properties and applications. Gazi University Journal of Science, 33(1): 265-277.

Eghwerido J, Zelibe S, Efe-Eyefia E (2020a). Gompertz-alpha power iverted exponential distribution: Properties and applications. Thailand Statistician, 18(3): 319-332.

Eghwerido JT, Ikwuoche JD, Adubisi OD (2020b). Inverse odd Weibull generated family of distribution. Pakistan Journal of Statistics and Operation Research, 16(3): 617-633.

Eghwerido JT, Lawrence N, Adubisi OD (2020). The Gompertz extended generalized exponential 
distribution: Properties and applications. Communications Faculty of Sciences University of Ankara Series A1 Mathematics and Statistics, 69(1): 739-753.

Eghwerido JT, Nzei LC, Agu FI (2020). The alpha power Gompertz distribution: Characterization, properties, and applications. Sankhya A. Forthcoming, https://doi.org/10.1007/ s13171-020-00198-0.

El-Damcese M, Mustafa A, El-Desouky B, Mustafa M (2015). The odd generalized exponential Gompertz. Applied Mathematics, 6: 2340-2353.

El-Gohary A, Alshamrani A, Al-Otaibi AN (2013). The generalized Gompertz distribution. Applied Mathematical Modelling, 37(1): 13-24.

Elbatal I, Jamal F, Chesneau C, Elgarh M, Alrajhi S (2019). The modified Beta Gompertz distribution: Theory and applications. Mathematics, 7(1): 3.

Gompertz B (1825). On the nature of the function expressive of the law of human mortality, and on a new mode of determining the value of life contingencies. Philosophical Transactions of the Royal Society of London, (115): 513-583.

Khan M, King R, Hudson I (2017). Transmuted generalized Gompertz distribution with application. Journal of Statistical Theory and Applications, 16: 65-80.

Mahdavi A, Kundu D (2017). A new method for generating distributions with an application to exponential distribution. Communications in Statistics-Theory and Methods, 46(13): 65436557.

Marshall AW, Olkin I (1997). A new method for adding a parameter to a family of distributions with application to the exponential and Weibull families. Biometrika, 84(3): 641-652.

Mohamed EM, Gauss MC, Ahmed ZA, Hazem A (2019). The alpha power transformation family: Properties and applications. Pakistan Journal of Statistics and Operation Research, 15(3): 525-545.

Nichols MD, Padgett WJ (2006). A bootstrap control chart for Weibull percentiles. Quality and Reliability Engineering International, 22(2): 141-151.

Smith RL, Naylor JC (1987). A comparison of maximum likelihood and Bayesian estimators for the three-parameter Weibull distribution. Journal of the Royal Statistical Society: Series C (Applied Statistics), 36(3): 358-369.

Tjørve KM, Tjørve E (2017). The use of Gompertz models in growth analyses, and new Gompertz-model approach: An addition to the Unified-Richards family. PLOS ONE, 12(6): e0178691.

Zelibe SC, Eghwerido JT, Efe-Eyefia E (2019). Kumaraswamy-alpha power inverted exponential distribution: Properties and applications. Istatistik Journal of the Turkish Statistical Association, 12(2): 35-48. 